\title{
Effects of Amino Acids on the Utilization of Tryptophan and Indole for Growth by a Mutant of Neurospora crassa
}

\author{
By H. E. BROCKMAN* \\ Department of Biological Sciences, Florida State University, Tallahassee, \\ Florida, and Biology Division, Oak Ridge National Laboratory, \\ Oak Ridge, Tennessee, U.S.A.
}

(Received 13 May 1963)

\begin{abstract}
SUMMARY
Fourteen of twenty-one naturally occurring amino acids inhibited competitively the utilization of tryptophan for growth by an indole- or tryptophan-requiring strain of Neurospora crassa. At less than inhibitory ratios of amino acid to tryptophan, certain amino acids enhanced the growth of this mutant. Growth on indole was neither enhanced nor inhibited by amino acids other than tryptophan. The uptake of tryptophan from the medium was inhibited by phenylalanine. The effects of amino acid on the growth of this strain with tryptophan can be explained by an inhibition of tryptophan uptake from the medium.
\end{abstract}

\section{INTRODUCTION}

Various naturally occurring amino acids can inhibit the growth of certain amino acid-requiring mutants of Neurospora crassa. A mutant which grew on isoleucine + valine was inhibited by phenylalanine, norleucine or norvaline (Bonner, Tatum \& Beadle, 1943); the growth of a lysine-requiring mutant was competitively inhibited by arginine (Doermann, 1944), and arginine-deficient mutants were competitively inhibited by lysine (Srb, 1953). Lein, Mitchell \& Houlahan (1948) noted that three types of Neurospora mutants-histidine-, tryptophan- and methionine-requiringdid not grow on a complete medium. The inhibition of histidine-requiring mutants by the complete medium was later shown to be due to certain mixtures of amino acids (Haas, Mitchell, Ames \& Mitchell, 1952); leucine, isoleucine, valine, methionine, glycine, tyrosine, phenylalanine, tryptophan or histamine were inhibitory in combination with lysine or arginine. Mathieson \& Catcheside (1955) showed that the two combinations, arginine + methionine and arginine + tryptophan, were competitive with histidine; they also concluded that amino acids inhibit the growth of histidine-requiring mutants by preventing histidine uptake into the mycelium. Norvaline, tyrosine or $\alpha$-aminobutyric acid inhibited the growth of a mutant which grew maximally on methionine + threonine (Teas, Horowitz \& Fling, 1948).

Haddox (1952) reported that the growth of a tyrosine-requiring mutant was inhibited by leucine and that a mutant which required phenylalanine or tyrosine was inhibited by leucine or tryptophan. The growth of another tyrosine-requiring mutant (T-145) on tyrosine was inhibited by 18 of 23 amino acids tested (DeBusk \&

* Present address: Department of Biological Sciences, Illinois State University, Normal, Illinois. 
Wagner, 1953). A mutant which grew on any one of the intermediates between indole and nicotinic acid was inhibited by 12 of 21 amino acids that were tested in combination with tryptophan, kynurenine or 3-hydroxykynurenine (Shanmugasundaram \& Sarma, 1954; Panicker, Shanmugasundaram \& Acharya, 1962). Soboren \& Nyc (1961) reported that 4 of 17 naturally occurring amino acids inhibited growth of the tryptophan-requiring strain c-83. Brockman, DeBusk \& Wagner (1959) showed that the utilization of phenylalanine for growth by a phenylalanine- or phenylpyruvate-requiring mutant (E-5212) was competitively inhibited by any one of 14 naturally occurring amino acids. The utilization of phenylpyruvate, the $\alpha$-keto analogue of phenylalanine, for growth by this strain was not inhibited by amino acids but was competitively inhibited by other $\alpha$-keto acids. Therefore $\alpha$-amino acids may be antagonists to amino acid utilization for growth in this mutant, and $\alpha$-keto acids may inhibit keto acid utilization. The importance of the group at the $\alpha$-position in determining inhibition specificity was further demonstrated by the observation that $\alpha$-hydroxyisovalerate (the $\alpha$-hydroxy analogue of valine) did not completely inhibit either phenylalanine or phenylpyruvate utilization for growth (Brockman, 1960).

The effects of amino acids on the growth of two aromatic amino acid-requiring mutants (T-145 and E-5212) of Neurospora were studied by DeBusk \& Wagner (1953), Brockman et al. (1959) and Brockman (1960). The present report extends the observations to the indole- or tryptophan-requiring strain Fs-108. This was done with the following objectives: $(a)$ to determine whether the same family of inhibitory amino acids would be found with strain Fs-108 as was found with strains E-5212 and T-145; $(b)$ to see whether the utilization of indole for growth by strain FS-108 would be unaffected by amino acids as keto acid utilization was in strains E-5212 and $\mathrm{T}-145 ;(c)$ to investigate the phenomenon of sparing of the tryptophan requirement by low concentrations of certain amino acids that had been observed in an early experiment with strain FS-108; $(d)$ to determine the relation, if any, between the phenomena of amino acid sparing and inhibition. A preliminary report has been given (Brockman \& DeBusk, 1960).

\section{METHODS}

The mutant strain FS-108 of Neurospora crassa was recovered by the author from a filtration-concentration experiment (Woodward, De Zeeuw \& Srb, 1954) following ultraviolet irradiation of the St Lawrence wild-type strain sT-74 A. Its growth requirement is satisfied only by indole or tryptophan, and the gene governing this requirement is located at the tryp-1 locus of linkage group IIIR. Vegetative cultures were maintained on slopes of complete medium + tryptophan. Since the growth of strain Fs-108 is inhibited by the amino acids in complete media, a 'weak' complete medium consisting of $2 \%$ glycerol, $1.5 \%$ agar, $0.05 \%$ liver fraction ' $L$ ' (Nutritional Biochemicals Corp., Cleveland 28, Ohio), $0.05 \%$ yeast extract and $\mathbf{0 . 0 5} \%$ malt extract (Difco Laboratories, Detroit 1, Michigan) in Fries minimal medium was used.

Growth assays were done in $125 \mathrm{ml}$. Erlenmeyer flasks containing $25 \mathrm{ml}$. liquid Fries minimal medium (Beadle \& Tatum, 1945). The flasks were inoculated with one to two drops of just visibly turbid conidial suspension and incubated for 3 days 
at about $27^{\circ}$ unless noted otherwise. The mycelial growth was harvested from each flask with a glass hook and excess medium removed by pressing the mycelial pad on a paper towel. These pads were dried overnight at $60^{\circ}$ before weighing. Each weight value quoted is an average from duplicate flasks recorded to the nearest milligram; pads weighing less than $5 \mathrm{mg}$. were recorded as zero. Tryptophan was assayed colorimetrically by the method of Nason, Kaplan \& Colowick (1951).

\section{RESULTS}

Many metabolites, including the known intermediates of aromatic amino acid biosynthesis, were tested for growth-promoting activity for the Neurospora crassa mutant strain Fs-108; and only indole or tryptophan was active. Characteristic growth response curves (Fig. 1) showed that, although the mutant responded better to indole than to tryptophan at low concentrations, the maximum growth achieved in 3 days was nearly identical. The inhibition by indole at concentrations greater than $4 \mu \mathrm{mole} / 25 \mathrm{ml}$. is not unique for strain Fs-108, since indole inhibition has also been observed in wild-type strains (Cushing, Schwartz \& Bennet, 1949), adeninerequiring mutants (Lein et al. 1948) and tryptophan- or indole-requiring mutants (Newmeyer \& Tatum, 1953) of $N$. crassa.

\section{Table 1. Competitive inhibition of tryptophan utilization for growth in the Neurospora mutant strain Fs-108}

At each $\mu$ mole value of $L$-tryptophan, the concentration of inhibitory amino acid was varied to give the desired ratio of inhibitory amino acid to tryptophan. The dry weight values were determined by the methods described in Fig. 1.

$\mu$ mole L-tryptophan/flask

\begin{tabular}{|c|c|c|c|c|c|c|c|}
\hline \multirow{3}{*}{$\begin{array}{l}\text { Inhibitory } \\
\text { amino acid }\end{array}$} & \multirow{3}{*}{$\begin{array}{l}\text { Ratio of inhibitory } \\
\text { amino acid : L-tryptophan }\end{array}$} & & & & & & \multirow{3}{*}{32} \\
\hline & & 1 & $\mathbf{2}$ & 4 & 8 & 16 & \\
\hline & & \multicolumn{5}{|c|}{ Growth (mg. dry wt.) } & \\
\hline None & 一 & 14 & 25 & 43 & $\mathbf{5 5}$ & 51 & 42 \\
\hline \multirow[t]{2}{*}{ L-Phenylalanine } & 10 & 42 & $\mathbf{5 2}$ & 70 & 68 & 65 & 61 \\
\hline & 20 & $\mathbf{0}$ & $\mathbf{0}$ & $\mathbf{0}$ & $\mathbf{0}$ & $\mathbf{0}$ & $\mathbf{0}$ \\
\hline \multirow[t]{3}{*}{ L-Leucine } & 20 & $\mathbf{3 9}$ & 45 & $\mathbf{5 1}$ & 49 & 51 & 51 \\
\hline & $\mathbf{3 0}$ & 26 & $\mathbf{3 8}$ & $\mathbf{3 3}$ & 27 & 27 & 18 \\
\hline & 40 & $\mathbf{0}$ & $\mathbf{0}$ & $\mathbf{0}$ & $\mathbf{0}$ & $\mathbf{0}$ & $\mathbf{0}$ \\
\hline \multirow[t]{3}{*}{ L-Valine } & 130 & 16 & 32 & $\mathbf{3 0}$ & 22 & 12 & - \\
\hline & 140 & 11 & 10 & 25 & 14 & 5 & 一 \\
\hline & 150 & $\mathbf{0}$ & $\mathbf{0}$ & $\mathbf{0}$ & $\mathbf{0}$ & $\mathbf{0}$ & - \\
\hline
\end{tabular}

Fourteen of 21 naturally occurring amino acids inhibited the growth of strain Fs-108 on L-tryptophan. The inhibition by each of these 14 amino acids was competitive in nature; the inhibition was dependent on the ratio of inhibitory amino acid to tryptophan, rather than on the absolute concentration of inhibitory amino acid. The results with three representative inhibitory amino acids are given in Table 1. The ratio of inhibitory amino acid to tryptophan which resulted in a complete inhibition of growth for 3 days has been defined as the inhibition index for that particular amino acid. The inhibition indices of all the amino acids tested are summarized in Table 2. Growth of the wild-type strain ST-74 A was not inhibited 


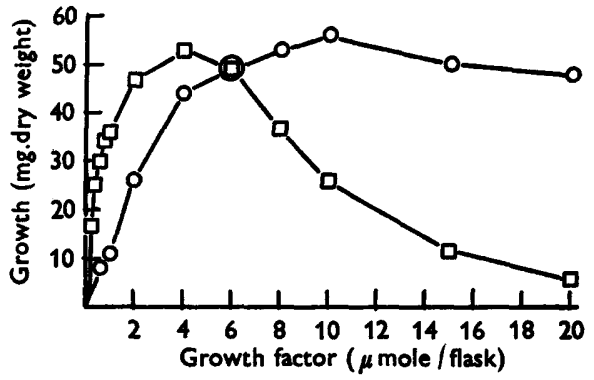

Fig. 1

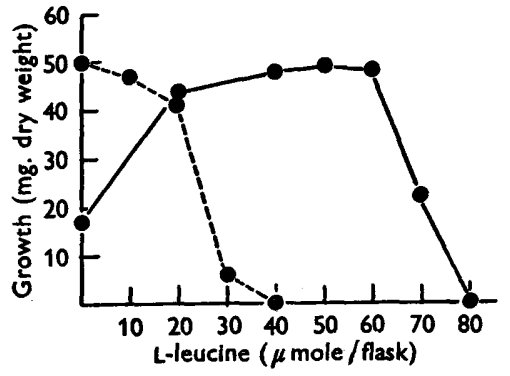

Fig. 2

Fig. 1. Growth response of the tryptophan- or indole-requiring mutant of Neurospora crassa strain Fs-108. The dry wt. of the mycelium was determined from duplicate $125 \mathrm{ml}$. Erlenmeyer flasks containing $25 \mathrm{mI}$. Fries minimal medium following 3 days static incubation at about $27^{\circ}, \square-\square$ indole; $O-O$ L-tryptophan.

Fig. 2. Additive nature of two inhibitory amino acids on the growth of Neurospora strain FS-108. - $2 \mu$ mole L-tryptophan/flask; --- $2 \mu$ mole L-tryptophan $+20 \mu$ mole L-phenylalanine/flask, a ratio of phenylalanine:tryptophan which enhances the growth over that given by tryptophan alone.

\section{Table 2. Amino acid inhibition indices for the Neurospora mutant strain Fs-108}

The inhibition index is defined as the ratio of inhibitory amino acid to L-tryptophan which results in a complete inhibition of growth for 3 days. Those amino acids which were not completely inhibitory of growth at a ratio of 500:1 were classified as non-inhibitory.

\begin{tabular}{|c|c|c|}
\hline $\begin{array}{c}\text { Inhibition } \\
\text { index }\end{array}$ & \multicolumn{2}{|c|}{ Amino acid } \\
\hline 10-20 & L-Phenylalanine & - \\
\hline $30-40$ & L-Leucine & • \\
\hline $50-60$ & L-Methionine & DL-Norvaline \\
\hline $60-70$ & L-Tyrosine & DL- $\alpha$-Aminobutyric acid \\
\hline $80-90$ & DL-Norleucine & . \\
\hline $120-130$ & $\mathrm{~L}$-Alanine & . \\
\hline $140-150$ & L-Valine & - \\
\hline 300-325 & Glycine & DL-Threonine \\
\hline 325-350 & DL-Serine & DL-Isoleucine \\
\hline $350-375$ & DL-Asparagine & . \\
\hline Non-inhibitory & L-Lysine & L-Glutamic acid \\
\hline & L-Arginine & L-Aspartic acid \\
\hline & L-Proline & L-Glutamine \\
\hline & L-Histidine & . \\
\hline
\end{tabular}

by concentrations of the individual amino acids equal to those tested for strain Fs-108.

From Table 1 it is evident that less than inhibitory concentrations of certain inhibitory amino acids, such as phenylalanine, enhanced the growth of strain Fs-108 over that on tryptophan alone. To test further this stimulatory action of the amino acids on the tryptophan requirement of strain FS-108, two concentrations of each of the amino acids were superimposed on $1 \mu$ mole of tryptophan (Table 3 ); the amino acids are tabulated in the order of increasing inhibition index. By referring to the column in which $\mathbf{1 0} \mu$ mole of each amino acid was tested, it is evident that, 
in general, as the inhibition index increased the degree of stimulation decreased. Amino acids with inhibition indices greater than 300 enhanced growth very little, and little or no stimulation was observed with $10 \mu$ mole of the non-inhibitory amino acids. Some of the amino acids which were not stimulatory at the $10 \mu$ mole concentration did spare the tryptophan requirement when tested at one-half the concentration of the inhibition index. None of the amino acids supported growth of strain FS-108 in the absence of tryptophan.

Table 3. Stimulation of the growth of Neurospora strain Fs-108 on tryptophan by certain amino acids

One $\mu$ mole L-tryptophan, which induced $10 \mathrm{mg}$. dry wt. growth in 3 days, was added to all growth flasks. The number of $\mu$ mole of inhibitory amino acid equivalent to one-half the inhibition index was calculated from Table 2, and in the case of the non-inhibitory amino acids $250 \mu$ mole was tested.

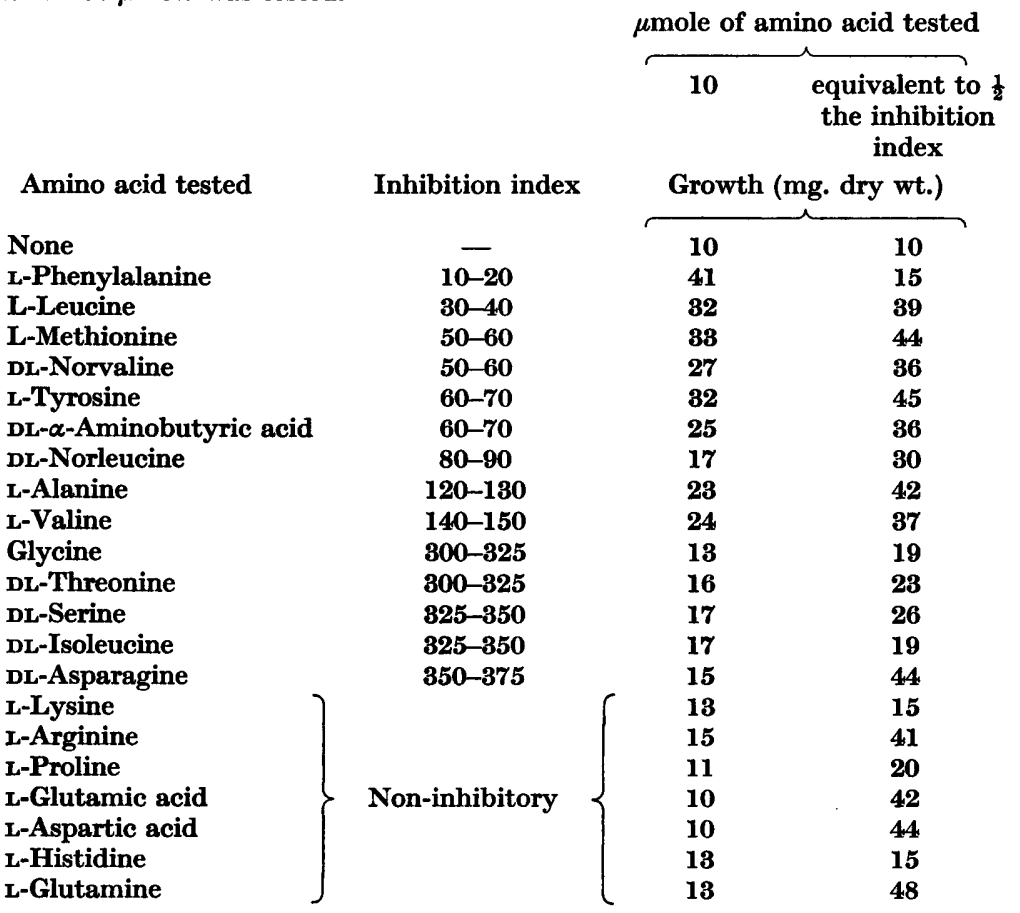

Bergmann, Sicher \& Volcani (1952) reported that $p$-aminobenzoic acid (PABA) inhibition of a tryptophan-requiring Escherichia coli strain was competitively annulled by tryptophan, and that phenylalanine and tyrosine, in addition to sparing the tryptophan requirement, annulled the PABA inhibition of tryptophan utilization. In relation to the sparing of the tryptophan requirement in strain Fs-108, it was of interest to determine whether inhibition of the tryptophan utilization by a specific amino acid would be annulled by stimulatory concentrations of a second amino acid. This possibility was tested, as shown in Fig. 2. If phenylalanine had annulled the leucine inhibition of tryptophan utilization, more leucine would have been required for complete inhibition of growth in the presence of phenylalanine + tryptophan than in the presence of tryptophan alone. On the contrary, the inhibition 
index was less when the initial growth was enhanced by a sparing concentration of phenylalanine, and, furthermore, the results suggest that phenylalanine and leucine are strictly additive in their inhibitory action on tryptophan utilization for growth of strain Fs-108.

Since certain amino acids had a marked influence on tryptophan utilization for growth by strain FS-108, all 21 amino acids were retested for their ability to inhibit or spare indole utilization. Either 250 or $500 \mu$ mole of each of the 21 amino acids in combination with $1 \mu$ mole indole did not generally result in a significantly different growth value from that given on indole alone. Five hundred $\mu$ mole of glycine, DL-serine, L-histidine, DL-norvaline or DL- $\alpha$-aminobutyric acid induced a $50 \%$

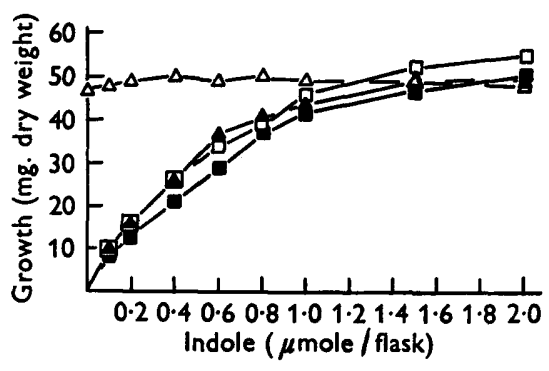

Fig. 3

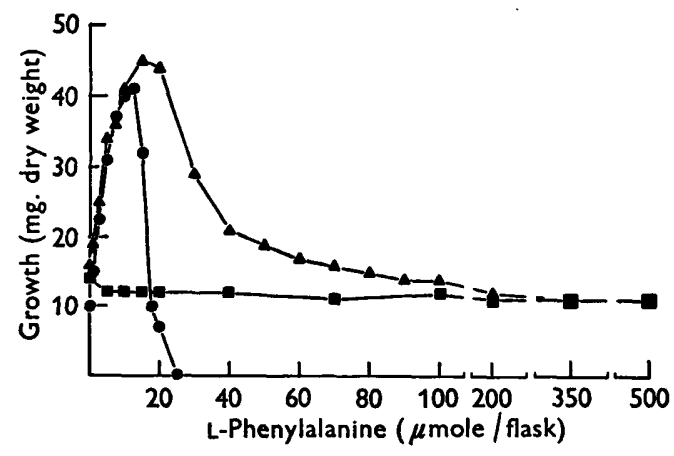

Fig. 4

Fig. 3. Indole annulment of phenylalanine inhibition of growth in the Neurospora strain Fs-108. $\square-\square$ indole only; $\square-\square$ indole $+500 \mu$ mole L-phenylalanine/flask; $\triangle-\triangle$ indole $+5 \mu$ mole $L$-tryptophan/flask; $\Delta-\Delta$ indole $+5 \mu$ mole $L$-tryptophan and $500 \mu$ mole L-phenylalanine/flask.

Fig. 4. Effect of L-phenylalanine on the growth of Neurospora strain Fs-108 under three different nutritional conditions. $-0.2 \mu$ mole indole/flask; $0-1 \mu$ mole L-tryptophan/flask; $\Delta-\Delta 1 \mu$ mole L-tryptophan $+0 \cdot 2 \mu$ mole indole/flask.

growth inhibition, but further tests showed that the antagonisms were not competitive. Various keto acids, vitamins, organic acids, purines and pyrimidines were also tested in combination with indole, but to date no naturally occurring metabolite has been found which will competitively inhibit utilization of indole for growth.

It was further found that indole annulled non-competitively all the amino acid inhibitions of tryptophan utilization. Indole relief of phenylalanine inhibition of tryptophan utilization was studied in detail (Fig. 3). When various concentrations of indole were added to flasks containing $5 \mu$ mole tryptophan $+500 \mu$ mole phenylalanine, the resulting growth curve was always nearly identical with the control curves of growth on indole alone or on indole + phenylalanine. These results suggest that only indole, and not tryptophan, is utilized for growth by strain Fs-108 when indole annuls the phenylalanine inhibition of tryptophan utilization. Other compounds involved in tryptophan metabolism, including 3-hydroxyanthranilic acid, kynurenine and nicotinic acid, were tested for annulment of amino acid inhibition of tryptophan utilization, but only indole was effective.

It is clear from the data so far presented that the growth response of strain Fs-108 in the presence of an amino acid other than tryptophan was markedly dependent 
on the growth requirement that was supplied: either tryptophan, indole, or indole + tryptophan. To show better the growth responses of strain Fs-108 to an inhibitory amino acid, various concentrations of phenylalanine were superimposed on the three possible growth supplements of strain Fs-108 (Fig. 4). Growth on indole was neither enhanced nor depressed at any concentration of phenylalanine, except for an initial small decrease. In contrast, growth on tryptophan was both enhanced and inhibited by phenylalanine over a $30 \mu$ mole concentration range. In the presence of tryptophan + indole, the growth requirement was again markedly spared, and growth was slowly decreased to $12 \mathrm{mg}$. dry wt. at very high concentrations of phenylalanine. From Fig. 4 it cannot be determined whether this $12 \mathrm{mg}$. dry wt. value was equivalent to the growth given on indole or tryptophan, but other experiments (as in Fig. 3) showed that growth was always decreased to a value which would have been obtained on indole alone.

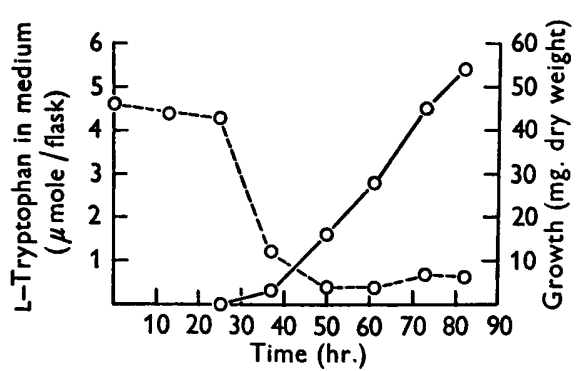

Fig. 5

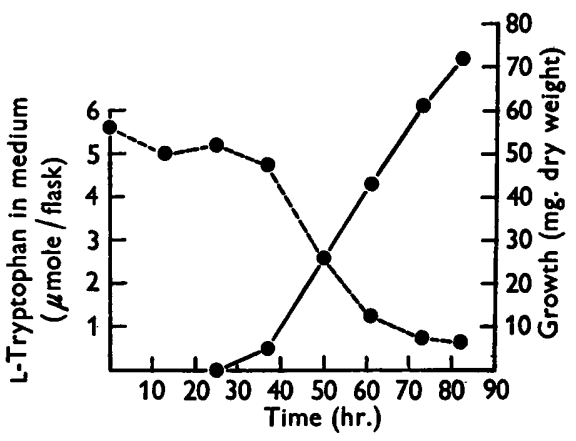

Fig. 6

Fig. 5. Relation between tryptophan uptake and growth in Neurospora strain Fs-108 in the absence of inhibitory amino acid. Flasks containing $25 \mathrm{ml}$. of Fries minimal medium $+5 \mu$ mole L-tryptophan were inoculated with $7 \cdot 45 \times 10^{4}$ conidia/flask. At each indicated time, the tryptophan remaining in the culture fluid was determined colorimetrically by the method of Nason et al. (1951) following centrifugation of the conidia and/or mycelium; dry wt. of mycelium was also recorded. $\bigcirc---\bigcirc$ L-tryptophan; $\bigcirc-O$ growth.

Fig. 6. Relation between tryptophan uptake and growth of Neurospora strain Fs-108 in the presence of a sparing concentration of the inhibitory amino acid, c-phenylalanine. Experimental conditions were the same as in Fig. 5 except that $50 \mu$ mole of L-phenylalanine were added to each flask. --. L-tryptophan; - growth.

The disappearance of tryptophan from the medium during a growth period of 3.5 days was measured colorimetrically by the method of Nason et al. (1951). Flasks containing tryptophan, tryptophan + a sparing concentration of phenylalanine, and tryptophan + an inhibitory concentration of phenylalanine, were inoculated with $7 \cdot 45 \times 10^{4}$ conidia/flask. About every $12 \mathrm{hr}$ the contents of duplicate flasks of each type were centrifuged and a sample of the culture fluid assayed for tryptophan; growth (dry wt.) was measured simultaneously. Tryptophan uptake from the medium and the resulting growth in the absence of inhibitory amino acid during incubation for 3.5 days are given in Fig. 5. Fig. 6 shows the results when a sparing concentration of phenylalanine was added. Tryptophan was taken up at a very rapid rate between 24 and $36 \mathrm{hr}$. in the absence of inhibitor (Fig. 5), but a sparing concentration of phenylalanine decreased this rate of uptake significantly (Fig. 6). No growth or tryptophan uptake from the medium occurred 
during the 3.5 days when an inhibitory concentration of phenylalanine was used (250 $\mu$ mole phenylalanine $+5 \mu$ mole tryptophan). A comparison of Figs. 5 and 6 leads to the surprising conclusion that the growth of strain Fs-108 was enhanced by the sparing concentration of phenylalanine even though the uptake of its requirement, tryptophan, was inhibited.

These results led to the formulation of the following hypothesis. In the absence of inhibitory amino acids strain Fs-108 transports tryptophan into the organism at a rate greater than that necessary for optimum growth. This accumulation of tryptophan might result in an intracellular imbalance of amino acids (Soboren \& Nyc, 1961) unfavourable for growth, or the accumulated tryptophan might be degraded before it could be utilized for growth (Haskins \& Mitchell, 1949). Inhibitory amino acids are antagonists to the transport of tryptophan into the cell, and a certain ratio of inhibitory amino acid to tryptophan produces an optimum rate of tryptophan uptake; this ratio is the one which enhances growth most. As the ratio of inhibitory amino acid to tryptophan is further increased, the decreased rate of tryptophan uptake becomes limiting for growth, and complete growth inhibition eventually occurs. According to this hypothesis, the enhancement of growth by sparing concentrations of an inhibitory amino acid is due solely to its regulatory effect on the rate of tryptophan uptake and is not the result of other, unspecified, intracellular functions.

\section{Table 4. Fractional addition of tryptophan to Neurospora strain Fs-108}

Growth in mg. dry wt. was measured $72 \mathrm{hr}$ after the flasks were inoculated. The types of L-tryptophan addition were $(a) 1 \mu$ mole or $5 \mu$ mole added at the time of inoculation; (b) $0.2 \mu$ mole added at the time of inoculation and 12, 24, 36 and $48 \mathrm{hr}$ later for a total of $1 \mu$ mole, or $1 \mu$ mole added at inoculation time and at the same subsequent times for a total of $5 \mu$ mole.

$$
\text { Type of tryptophan addition }
$$

$\begin{array}{ccc}\begin{array}{c}\mu \text { mole } \\ \text { L-tryptophan } \\ 1\end{array} & \overbrace{11}^{(a)}(b) \\ 5 & 40 & 23 \\ \text { Growth (mg. dry wt.) }\end{array}$

To test this explanation of sparing, it was necessary to duplicate the action of sparing concentrations of an amino acid, i.e. to decrease the rate of tryptophan uptake in the absence of an inhibitory amino acid. This can be attempted most easily by adding small concentrations of tryptophan to an inoculated growth flask periodically throughout the 3-day incubation period. Table 4 shows the results of such an experiment. It is clear that addition of a total amount of 1 or $5 \mu$ mole tryptophan at zero time gave less growth than when those total amounts were added fractionally during the incubation period. Growth was not enhanced to the extent previously shown by sparing concentrations of certain amino acids, but growth could probably be further increased by adding the tryptophan more frequently in even smaller fractions. 


\section{DISCUSSION}

The ability of certain naturally occurring amino acids to inhibit competitively utilization of the amino acid required for growth and the relative effectiveness for inhibition are similar in three aromatic amino acid-requiring mutants of Neurospora crassa: tyrosine-deficient strain T-145 (DeBusk \& Wagner, 1953), phenylalaninedeficient strain E-5212 (Brockman et al. 1959), and tryptophan-deficient strain FS-108 (this report). In most previous publications, only a limited number of amino acids were reported as inhibitory for a given amino acid-requiring strain (see Introduction). With few exceptions, however, all of the common amino acids were not tested, or they were not tested at sufficiently high ratios of amino acid to required amino acid. Consequently, distinct relations between groups of inhibitory amino acids and classes of amino acid-deficient mutants are not readily apparent from a consideration of the previous literature. It does seem possible now to venture some predictions. Arginine and lysine are non-inhibitory in all aromatic amino acidrequiring mutants of Neurospora studied by the author and others, while argininedeficient mutants are inhibited by lysine and lysine-requiring mutants by arginine. It might be predicted, then, that glutamic acid-requiring mutants would not be inhibited by any of the amino acids which inhibit aromatic amino acid-requiring mutants; they might, however, be inhibited by some of the amino acids which do not inhibit aromatic amino acid-requiring strains. On the other hand, leucinerequiring mutants might be characterized by the same classes of inhibitory and non-inhibitory amino acids as have been found for aromatic amino acid-requiring mutants. These predictions are based on the hypothesis that the amino acids which inhibit growth of aromatic amino acid-requiring mutants exert their antagonistic action at a common site. The additive effect of phenylalanine and leucine in the inhibition of tryptophan utilization for growth of strain Fs-108 supports this hypothesis. It would be of interest to screen for amino acid inhibition of representative amino acid-requiring mutants of Neurospora so that a complete picture could be formulated. Mutants which will grow on more than one amino acid would be especially useful. Neurospora mutants deficient in aminating ability (am mutants) do grow on any one of a number of amino acids. Fincham (1950) has shown that four amino acids which are non-inhibitory or weak inhibitors for strain Fs-108-glycine, histidine, serine, and threonine-inhibited am mutants when they were grown on glutamate but not when they were grown on alanine.

All the results indicate that the inhibitory amino acids prevent tryptophan utilization for growth by inhibiting the entry of tryptophan into the organism rather than at some intracellular site of tryptophan utilization. This is shown directly by the present experiments on tryptophan uptake and is supported by the fact that the amino acids are not inhibitory to the wild-type strain or to strain Fs-108 supplemented with indole. The utilization of endogenous tryptophan (that synthesized by wild-type from simple precursors or by strain Fs-108 from indole) for growth is not inhibited, but the utilization of exogenous tryptophan for growth by strain FS-108 is inhibited competitively. Mathieson \& Catcheside (1955) concluded that the uptake of histidine by histidine-requiring strains is inhibited by certain amino acids. Similarly, casein hydrolysate inhibited tryptophan uptake by the Neurospora mutant strain 39401 (Panicker et al. 1962). The data are consistent with the supposition 
that there is one site with specificity for the aromatic amino acids and those amino acids which inhibit aromatic amino acid utilization.

The amino acids which are non-inhibitory to aromatic amino acid-requiring mutants can enter the organism at another site or sites. Similarly, there can be other sites specific for indole and for certain $\alpha$-keto acids.

The stimulation of growth of amino acid-requiring strains of Neurospora by various amino acids has been reported by other authors (Shanmugasundaram \& Sarma, 1954; Mathieson \& Catcheside, 1955; Newmeyer \& Tatum, 1953; Soboren \& Nyc, 1961). The present report shows that the ratio of test amino acid to required amino acid determines whether the test amino acids stimulate (spare the tryptophan requirement) or inhibit the growth of strain Fs-108 on tryptophan. An explanation of the sparing phenomenon which depends on a competition between the required amino acid and inhibitory amino acids for an uptake site has been advanced above. It was proposed that the ratio which enhances growth over that obtained with tryptophan alone is one which allows the required amino acid to enter the growing mycelium at a rate which is optimal for growth. Generally it is assumed that a metabolite which has no growth-promoting activity for a given mutant, but which spares that mutant's requirement, does so because it is an essential metabolic product of the compound which the mutant requires. The large number of naturally occurring amino acids which can spare the tryptophan requirement of strain Fs-108, the inability of these same amino acids to spare the indole requirement, and the fact that an amino acid can either spare or inhibit depending on the ratio of amino acid to tryptophan, support the proposed hypothesis.

This report is prepared in part from a dissertation presented to the Graduate School of Florida State University in partial fulfilment of the requirements for the degree of Doctor of Philosophy. The author expresses his appreciation to Dr A. G. DeBusk for his constant interest and encouragement during this work.

Oak Ridge National Laboratory is operated by Union Carbide Corporation for the United States Atomic Energy Commission.

\section{REFERENCES}

Beadle, G. W. \& TATUM, E. L. (1945). Neurospora II. Methods of producing and detecting mutations concerned with nutritional requirements. Amer. J. Bot. 32, 678.

Bergmann, E. D., Sicher, S. \& Volcani, B. E. (1952). Sparing effects in the biosynthesis of the aromatic amino-acids in Escherichia coli. Biochim. biophys. Acta, 9, 220.

Bonner, D., Tatum, E. L. \& Beadle, G. W. (1943). The genetic control of biochemical reactions in Neurospora: A mutant strain requiring isoleucine and valine. Arch. Biochem. 3, 71.

BrockMAN, H. E. (1960). Inhibition analysis of aromatic amino acid-requiring mutants of Neurospora crassa. Dissertation, Florida State University, Tallahassee, Florida.

Brockman, H. E. \& DeBusk, A. G. (1960). Relationships between sparing and inhibition in a tryptophan-deficient mutant of Neurospora crassa. Ass. Southeastern Biologists Bull. $7,2$.

Brockman, H. E., DeBusk, A. G. \& Wagner, R. P. (1959). Mutant-associated antagonisms in Neurospora crassa. Arch. Biochem. 84, 455.

Cushing, J. E., Schwartz, M. \& Bennett, R. (1949). Altered responses of Neurospora crassa to inhibiting concentrations of indole. J. Bact. 58, 433. 
DeBusk, A. G. \& Wagner, R. P. (1953). $p$-Hydroxyphenylpyruvic acid function in Neurospora crassa. J. Amer. chem. Soc. 75, 5131.

Doermann, A. H. (1944). A lysineless mutant of Neurospora and its inhibition by arginine. Arch. Biochem. 5, 373.

Fincham, J. R. S. (1950). Mutant strains of Neurospora deficient in aminating ability. J. biol. Chem. 182, 61.

HaAs, F., Mitchell, M. B., Ames, B. N. \& Mitchell, H. K. (1952). A series of histidineless mutants of Neurospora crassa. Genetics, 37, 217 .

Haddox, C. H. (1952). The accumulation of $\alpha$-phenylglycine by mutants of Neurospora crassa stimulated by phenylalanine and tyrosine. Proc. nat. Acad. Sci., Wash. 38, 482.

Haskins, F. A. \& Mitchell, H. K. (1949). Evidence for a tryptophan cycle in Neurospora. Proc. nat. Acad. Sci., Wash. 35, 500.

Lein, J., Mrtchell, H. K. \& Houlahan, M. B. (1948). A method for selection of biochemical mutants of Neurospora. Proc. nat. Acad. Sci., Wash. 34, 435.

Mathieson, M. J. \& Catcheside, D. G. (1955). Inhibition of histidine uptake in Neurospora crassa. J. gen. Microbiol. $13,72$.

Nason, A., Kaplan, N. O. \& Colowick, S. P. (1951). Changes in enzymatic constitution in zinc-deficient Neurospora. J. biol. Chem. 188, 397.

NewmeYer, D. \& TAtum, E. L. (1953). Gene expression in Neurospora mutants requiring nicotinic acid or tryptophan. Amer. J. Bot. 40, 393.

Panicker, R. K., Shanmugasundaram, E. R. B. \& Acharya, U. S. (1962). The effect of amino acid on the uptake and utilization of tryptophan and other precursors of nicotinic acid by Neurospora crassa. J. gen. Microbiol. 28, 409.

Shanmugasundaram, E. R. B. \& Sarma, P. S. (1954). Tryptophan utilization by Neurospora crassa and the influence of pure amino acids. Current Sci. India, 23, 224.

Soboren, J. \& Nyc, J. F. (1961). Amino acid interactions in Neurospora crassa. J. Bact. 82, 20.

SRB, A. M. (1953). Shifts in heterocaryotic dominance relations effected by modifier genes in Neurospora. Genetics, 38, 694.

Teas, H. J., Honowitz, N. H. \& Fling, M. (1948). Homoserine as a precursor of threonine and methionine in Neurospora. J. biol. Chem. 172, 651.

Woodward, V. W., de ZeEUw, J. R. \& SRB, A. M. (1954). The separation and isolation of particular biochemical mutants of Neurospora by differential germination of conidia, followed by filtration and selective plating. Proc. nat. Acad. Sci., Wash. 40, 192. 